\title{
Vascular anomalies
}

\section{Jyotsna Murthy}

Department of Plastic Surgery, Sri Ramachandra Medical Center \& Research Institute, Porur, Chennai, India

Address for correspondence: Dr. Jyotsna Murthy, Department of Plastic Surgery, Sri Ramachandra Medical Center \& Research Institute, Porur, Chennai - 600 116, India. E-mail: jmurthy@satyam.net.in

\section{ABSTRACT}

Management of vascular anomalies is an emerging multidisciplinary, super-specialisation field involving several surgical, medical and radiological specialties. Over the years, development in this field has been limited because of complex nomenclature and lack of consensus on the best practice for treatment of some of the more complex vascular anomalies. It was only in 1996 that the International Society of the Study of Vascular Anomalies defined nomenclature for the anomalies and gave clear guidelines on management, allowing for improved clinical practices. As in all fields of clinical medicine, the correct diagnosis of the vascular anomalies is essential to choose the appropriate treatment. This paper gives clear guidelines for diagnosis, understanding of the anomalies and discusses their management.

\section{KEY WORDS}

Vascular Anomalies, Haemangiomas, Capillary malformation, Venous malformation, Lymphatic malformation, Arterial Malformation

\section{INTRODUCTION}

 he treatment of vasualr anomalies is a new and rapidly developing discipline that demands teamwork between the pediatrician, plastic surgeon, interventional radiologist, pediatric surgeon, otolaryngologist and pediatric oncologist. It is a truly interdisciplinary domain because no single specialist has sufficient knowledge and expertise to diagnose and treat vascular anomalies in all organ systems.

The patient with vascular anomalies becomes a "nomad" because of the complexity of treatment, limited outcome and lack of expertise available with individual doctors. Most vascular anomalies involve the skin, the largest organ of the body, and therefore most vascular anomalies are obvious at birth. For centuries, vascular birth marks were called by vernacular names derived from folk beliefs about their origin. Thus, for example, it was believed that these abnormalities occurred because the mother ate strawberries excessively, or she saw blood during pregnancy or had sex during menstruation! ${ }^{1}$

\section{BIOLOGICAL CLASSFICATION}

One of the major obstacles to progress in the management of vascular anomalies has been the confusion arising in nomenclature and lack of clear cut guidelines for best clinical practice. It was only in 1996 that the International Society of the Study of Vascular Anomalies which met in Rome ${ }^{2}$ issued a new uniform nomenclature [Table 1] thus laying the foundation for improved management.

A vascular anomaly can either be a haemangioma (the Greek nominative suffix "oma" means tumor or 
Table 1: Classification of Vascular Anomalies
TUMOURS

Haemangioma

Haemnagio-endotheliomas
Angiosarcoma
MALFORMATION

Slow Flow

Capillary (CM)

Lymphatic (LM)

Venous (VM)

Fast Flow

Arterial (AM)

Ateriovenous Fistulae (AVF)

Arteriovenus (AVM) hyperplasia.) or a malformation. ${ }^{3}$ The term haemangioma refers to a common tumor seen at infancy that exhibits rapid postnatal growth and slow regression during childhood. Vascular malformations are comprised of abnormally formed channels that are lined by quiescent endothelium, which may or may not be obvious at birth. They never regress (except occasional emptying of lymphatic malformation) and grow with the child. Combined vascular malformations are often associated with soft tissue and skeletal overgrowth and most are know by eponyms [Table 2].

The word congenital and acquired should be used with caution when vascular anomalies are described. The term congenital is used only when the lesion is present at birth.

\section{CLINICAL MANAGEMENT}

Once the nomenclature is clarified, the accuracy of diagnosis based on the medical history and physical examination is more than $90 \%$ accurate. In the remaining $10 \%$, where the diagnosis is not clear, the most helpful clinical test is a "biopsy". The various radiological investigations are preferably done only to define visceral involvement and just before any treatment is contemplated. For example, an arteriogram is done in patients with arterial malformation not for diagnosis, which is easily done clinically but to know the major feeder artery prior to embolisation. Unfortunately, more often than not, major emphasis has been put on radiological investigations rather than diagnosing the lesion clinically.

\section{INFANTILE HAMANGIOMA}

Haemangiomas occurs in skin of 4-10\% of Caucasian new born with a 3-5 fold greater incidence in females. Dark skinned infants have a lower incidence. Haemengiomas occurs most frequently in the head/neck region (60\%) followed by the trunk (25\%) and extremities (15\%). Eighty
Table 2: Combined vascular malformation associated with overgrowth

\section{Slow-flow}

Kipple-Trenaunay syndrome (CLVM)

Proteus syndrome (CLVM + lipomas and connective tissue tumors) Maffuci syndrome (VM+ endochondroma)

Fast-flow

Parkes Weber Syndrome (CAVM, VLAVM)

Bannayan-Railey-Ruvalcaba syndrome (AVM + delayed motor and speech development, proximal myopathy)

percent of tumors are single, $20 \%$ are multifocal. ${ }^{3}$ No details about incidence in the Indian population available. Multiple cutaneous tumors are often associated with visceral lesions, typically located in liver, gastrointestinal tract, lungs and brain. Hemangiomata involving skin the manifest as red, raised and bosselated swellings. However, the tumors, which begin in deep dermis or subcutaneous tissues, are covered with normal skin. These lesions are likely to be confused with vascular malformations. There is a rare subgroup of midline hemangiomas that is associated with structural anomalies like ocular deformities, persistent embryonic intra/extracranial arteries, hypoplasia/absence of carotid/vertebral vessels, coarctation and right-sided aortic arch, Dandy-Walker malformation, sternal clefting and lumbar spinal dysraphism (PHACE syndrome).

Haemangiomas expand rapidly during infancy. After one year, they slowly regress. The first sign of regression is a change of the bright crimson color to a patchy gray mantle. The tumor feels less tense. The involuting phase extends from 1 to 5-7 years. The rate of regression is unrelated to the appearance, cutaneous depth, gender, site or size of tumour. The involuting phase occurs in $50 \%$ in 5 years and $70 \%$ in 7 years. Near normal skin is restored in $50 \%$ of children with hemangioma. In the others the involved skin is damaged, exhibiting telangiectasias, crepe like laxity and yellowish discoloration or patches of scarring. If the tumour was once large and protuberant, there can be residual fibrofatty tissue and redundant skin.

There is a rare variant of these typical tumors, they are haemaniomas growing in utero and presenting as fullygrown lesions at birth. These are called congenital hemangiomas. The other variant is Kaposiform hemangioendothelioma (KHE) or tufted angioma (TA). These tumors are linked to Kasabach-Merritt coagulopathy. Unlike common haemagiomas of infancy, KHE has a predilection for the trunk, retroperitoneum 
and proximal extremities. KHE has a high mortality of $20 \%$ and unpredictable sensitivity to corticosteriod, interferon alfa and vincristine treatment. ${ }^{2}$

\section{Radiological Investigations}

These are done to know the visceral involvement and in extensive lesions to study the response to treatment. On ultrasound examination, it may be difficult to differentiate proliferating haemangiomas from arterial malformations because both have a fast flow of blood in the lesion. MRI is the gold standard but needs general anesthesia or sedation for children.

\section{Treatment}

Parents need to be reassured regarding these rapidly progressing tumors in an otherwise normal child. The small and uncomplicated haemangioma does not warrant any treatment. However, medical therapy with steroid is necessary in children who develop an ulcerated lesion, which happens in $5 \%$ of children. Local application with viscous lidocain $(2.5 \%)$ helps to control pain. Active intervention is also necessary for lesions of eyelids, ears or lip leading to partial obstruction. Eyelid lesions can distort the growing cornea producing astigmatic amblyopia. Subglottic hemangioma presents insidiously as biphasic stridor at 6-8 weeks. Large haemangiomas may destroy skin structure of large areas and may lead to cardiac failure.

Corticosteroids form the first line of treatment. If the lesion is less than $2.5 \mathrm{~cm}$ diameter intralesional steroids are recommended while systemic administration is needed for a lesion larger than $2.5 \mathrm{~cm}$. Prednisolone $(2-4$

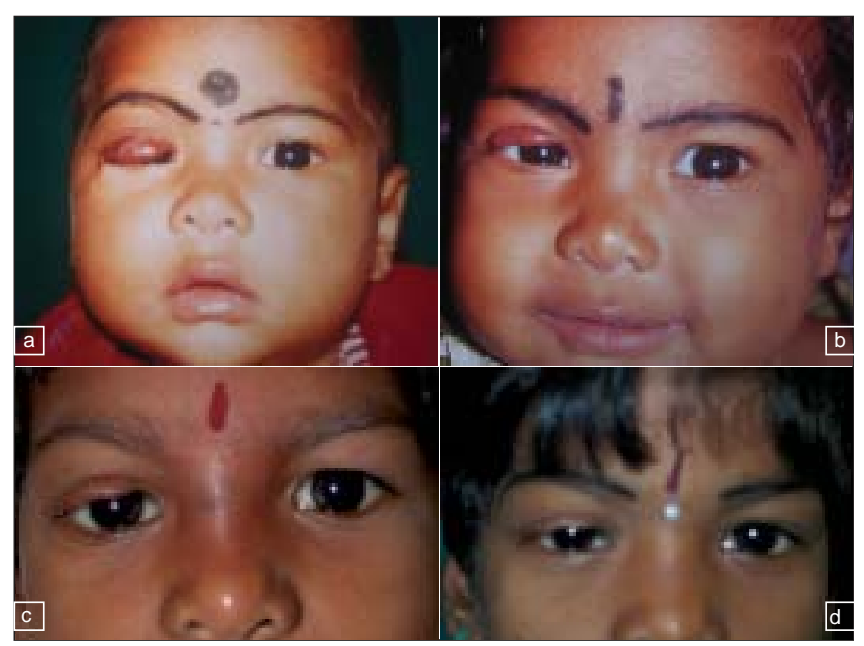

Figure 1: Upper lip haemangioma (a) treated by systemic steroid for 3 months (b). Cushingoid feature in immediate post therapy. Late result after 4 years (c and d). $\mathrm{mg} / \mathrm{kg} /$ day) is given as single dose till response is observed (for a total duration of 2-4 weeks). $80 \%$ lesions respond. ${ }^{4}$ Interferon alfa-2a is the second-line agent for management of life-threatening hemangiomas. Vincristin is a second-line treatment for problematic hemangiomas in infants which fail to respond to steroids, as well as in Kaposiform hemangioendothelioma.

Laser treatment does not play an important role in treatment of haemangioma except in the management of tiny telengiectasis that often remain in the involuted phase. Surgery is indicated in small, well-localized lesions of the eyelid, lip and neck or other parts of the body and when it is likely to lead to scar which is aesthetically more acceptable.

\section{VASCULAR MALFORMATIONS}

Vascular malformations are localized or diffuse errors of embryonic development. These anomalies are classified as capillary, lymphatic, venous, arterial or a combination of these depending on the clinical and histological appearance of the abnormal channels.

\section{Radiological Imaging}

The radiological imaging is done to establish the extent of the lesion and in some cases to confirm the clinical diagnosis. An experienced radiologist with knowledge in vascular anomalies is very essential for the diagnosis and management of these lesions. Simple X-rays assist in identifying the associated skeletal anomalies and presence of phleboliths clinch the diagnosis of venous malformation. Doppler studies help to differentiate slowflow from fast-flow anomalies as also the tumor mass of a haemangioma from anomalous channels of a vascular malformation.

MRI gives information about the extent, abnormal channels and flow characteristics of the vascular malformation. Capillary malformations are represented usually only as cutaneous thickening while venous malformations gives high signal intensity on T2-weighted images, and appear brighter than fatty tissue. Sometimes it is difficult to distinguish between venous, lymphatic and lympho-venous malformations. Lymphatic malformations are better delineated by administration of intravenous gadolinium and repetition of T1-weighted sequences. Magnetic resonance angiography and venography help before the planning treatment for 
arterio-venous malformations (AVM). Arteriography is the most invasive test and is rarely done for diagnosis. Rather, it is done only prior to embolisation of the AVM for treatment, palliation or prior to surgery.

\section{CAPILLARY MALFROMATIONS (CM)}

The most common capillary malformation observed clinically is the "port-wine stain". CMs are composed of dilated capillary-to-venular sized vessels, probably accounting for the darkening color and tendency to nodular ectasis. They can be localized or extensive, single or rarely multiple. The most common syndrome associated with CMs is Sturge-Weber Syndrome where $\mathrm{CM}$ occurs with ipsilateral ocular and leptomeningeal vascular anomalies. The tunable flash lamp pulsed-dye laser is the best way to treat these lesions. Facial and extremity CM may be associated skeletal and soft tissue hypertrophy, which may require surgical management. ${ }^{2}$

\section{LYMPHATIC MALFORMATION (LM)}

These are usually present at birth and most are evident before the age of 2 years. However, they can suddenly appear in older child or in adolescence. LM can be classified as microcystic (multiple small cyst less than 2.5 $\mathrm{cm}$ ) or macrocystic (large cysts $>2.5 \mathrm{~cm}$ ) or combined. They are most commonly located in axilla/chest, cervicofacial, mediastinum, retroperitoneum, buttock and anogenital areas. Apart from presenting as a tumour, LM can manifest as tiny vesicles on the skin. Intralesional bleeding is common and evident as tiny dark-red nodules.

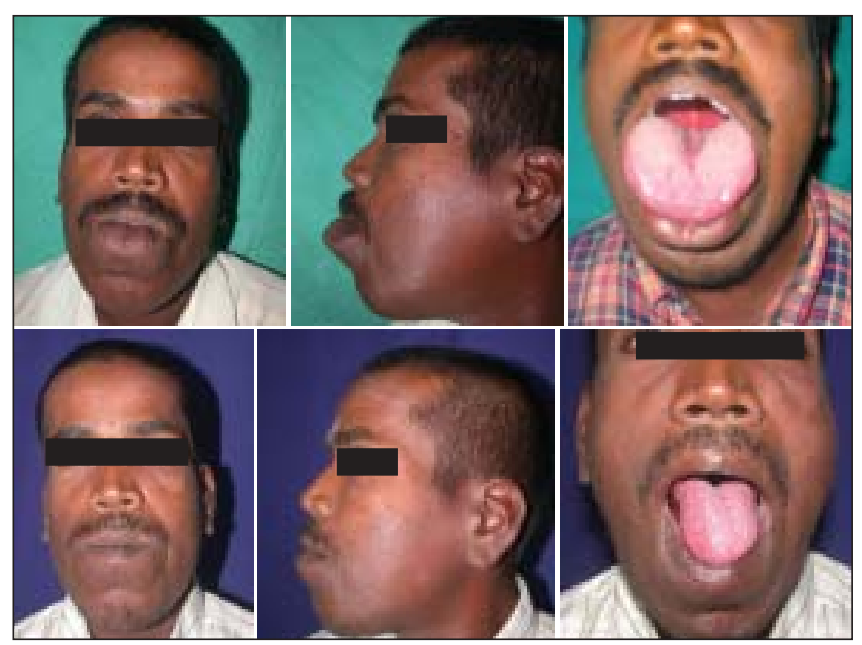

Figure 2: Capillary malformation with overgrowth of lower facial structures. Before (Top) and after reduction (Bottom) of lower lip and tongue. Will need skeletal work.
The diagnosis of LM is best confirmed by MRI where due to their high water content they are hyperintense on T2.

Management of repeated infections demands prompt action, often with intravenous antibiotics over 2-4 weeks. Resection is the only way to potentially "cure" LM. Often, staged excision is necessary and total excision is rarely possible. The surgeon should concentrate on a defined anatomic region and perform a thorough excision in that area. Macrocystic lesions are amenable to treatment with a sclerosing agent like absolute ethanol, doxycycline, sodium tetradecylsulfate or OK-432 (a killed strain of group-A streptococcus).

\section{VENOUS MALFORMATION (VM)}

Venous malformations are present at birth, although not always evident. They are bluish, soft and compressible and can be localized or extensive. They are usually present on the face, limbs or trunk. However, VM can also involve oro-nasopharynx, genitalia, bladder, brain, spinal cord, liver, spleen, lungs, skeletal muscles and bones. Patients often complain of pain and stiffness in the affected areas, especially upon awakening in the morning. Episodic thrombosis occurs and a phlebolith can appear as early as 2 years of age. VM can lead to obstructive symptoms in the oral cavity, oropharynx and larynx. VM of extremities can extend into joints, muscles and bones. An extremity VM rarely cause length discrepancy, although, the limb may be slight under-grown due to disuse. MRI is the most informative radiological technique for VMs. They give off as a bright signal on T2, brighter

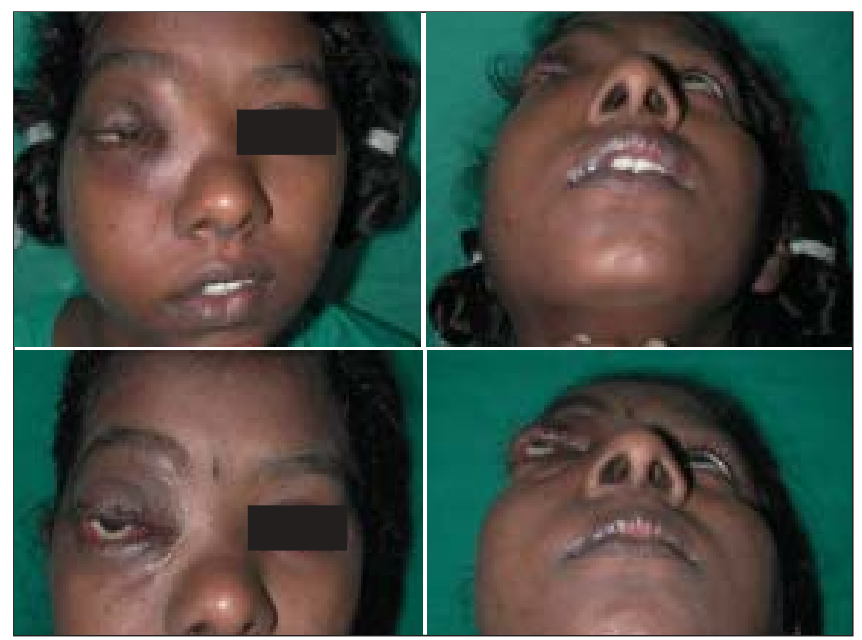

Figure 3: Lymphatic Malformation of Orbit, upper and lower eyelids, forehead and cheek. Preoperative (Top) and posteropative (bottom) photos after excision of LM in medical canthus and cheek and part of forehead. 


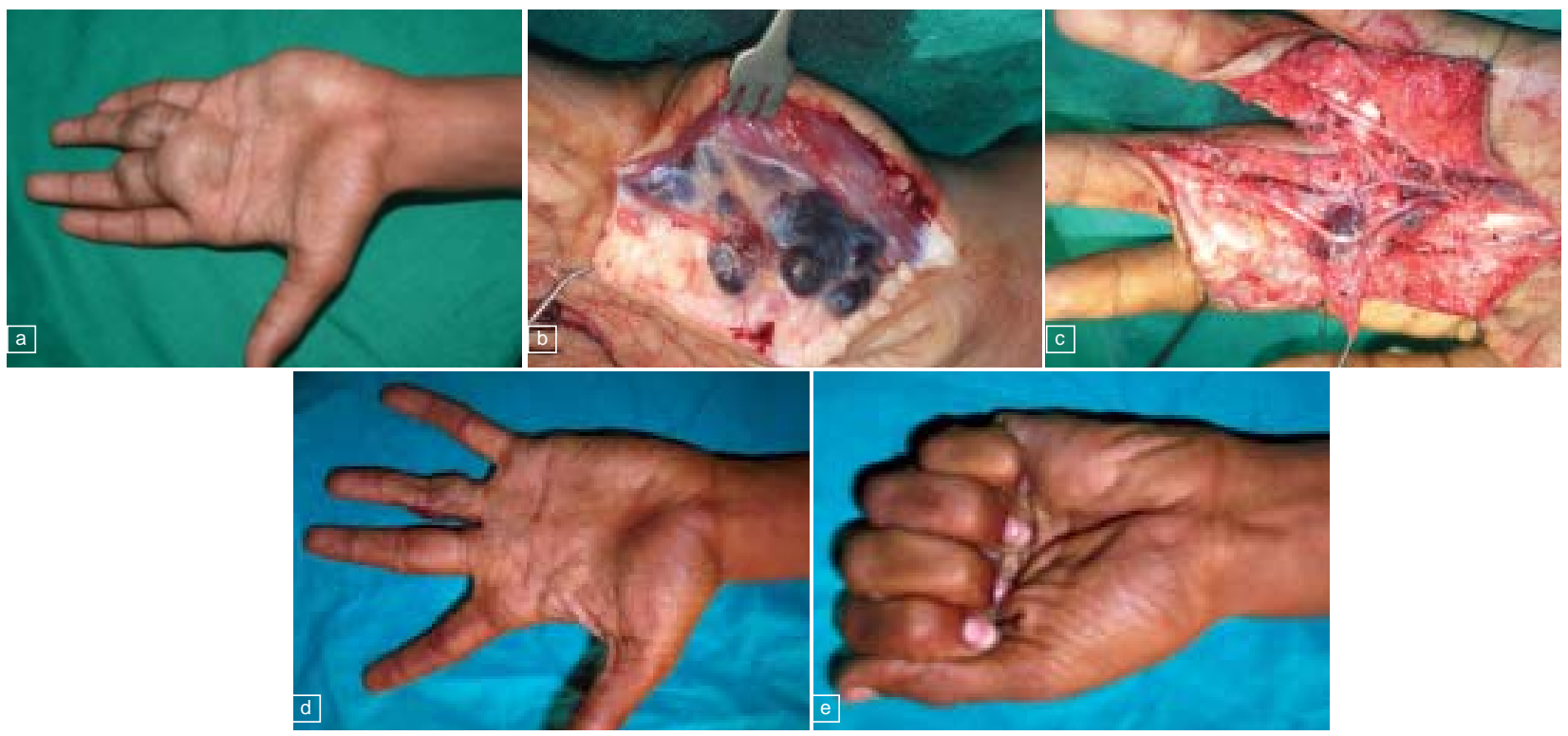

Figure 4: Venous malformation of hand: before (a), during (b, c) and after surgery (d, e)

than fat. A coagulation profile should be done on any child with large a VM as they are at risk for disseminated intravscular coagulopathy (DIC) following trauma or therapeutic intervention.

Treatment is indicated either for cosmetic or functional reasons. Small cutaneous VMs can be sclerosed with $1 \%$ sodium tetradecyl sulfate. A large cutaneus or intramuscular VM requires general anesthesia and "realtime" fluoroscopic monitoring during sclerotherapy. ${ }^{5}$ Absolute alcohol is the most effective sclerosant but should not be used near important structures like vessels and nerves, where $3 \%$ sodium tetradecylsulfate is preferred. Sclerotherapy of major VMs is dangerous and must be performed by a skilled and experienced interventional radiologist. ${ }^{6,7}$ Surgical excision of a residual VM may be warranted in some areas like face and extremities. VM of the gastrointestinal tract will need endoscopic sclerotherapy and resection. Surgical excision is the better option for $\mathrm{VM}$ in the distal parts of extremities (fingers and toes) due to the associated risk of development of gangrene.

\section{ARTERIAL MALFORMATION (AM)}

Intracranial arterial malformations are more common than extracranial. The extracranial arterial or arteriovenous malformations (AVM) are most commonly present in the head and neck region followed by limbs, trunk and viscera. AVMs are usually present at birth but are often given little attention because of their innocent appearance. They may appear like a red stain if superficial. Associated important clinical signs are warmth, and a "thrill" is palpable and a bruit can be heard over the lesion. Cutaneous consequences including ischemic changes, indolent ulceration, intractable pain and intermittent bleeding can sometimes occur as complications. As the AVM induces a high cardiac output state, decompensation and cardiac failure may set in.

Schobinger has described four clinical stages depending on the expansion and complications of AVM [Table 3]. ${ }^{8}$ MRI and Magnetic resonance angiography (MRA) best document the extent of the vascular malformation. Superselective angiography is usually not performed until intervention is scheduled.

Treatment is rarely indicated during infancy and early childhood. Once the diagnosis is made, the child should be followed up regularly. Intervention should be delayed until there are symptoms or signs, like ulceration, ischemic pain, bleeding or cardiac failure. ${ }^{8}$ Ligation or proximal emobilzation of the feeder vessels should never be performed because this causes rapid recruitment of flow from nearby arteries to supply the nidus which is

Table 3: Schobinger Clinical Staging system for AVMS

$\begin{array}{ll}\text { I: Quiescence } & \begin{array}{l}\text { Only Pink-bluishe stain and warmth } \\ \text { II: Expansion }\end{array} \\ & \begin{array}{l}\text { Enlarged swelling with pulsation, thrill and } \\ \text { bruit, veins are tense and tortuous } \\ \text { Same as above with ulceration, bleeding, pain } \\ \text { III : Destruction }\end{array} \\ \text { IV : Decompensation } & \begin{array}{l}\text { Same as stage III with cardiac failure } \\ \hline\end{array}\end{array}$




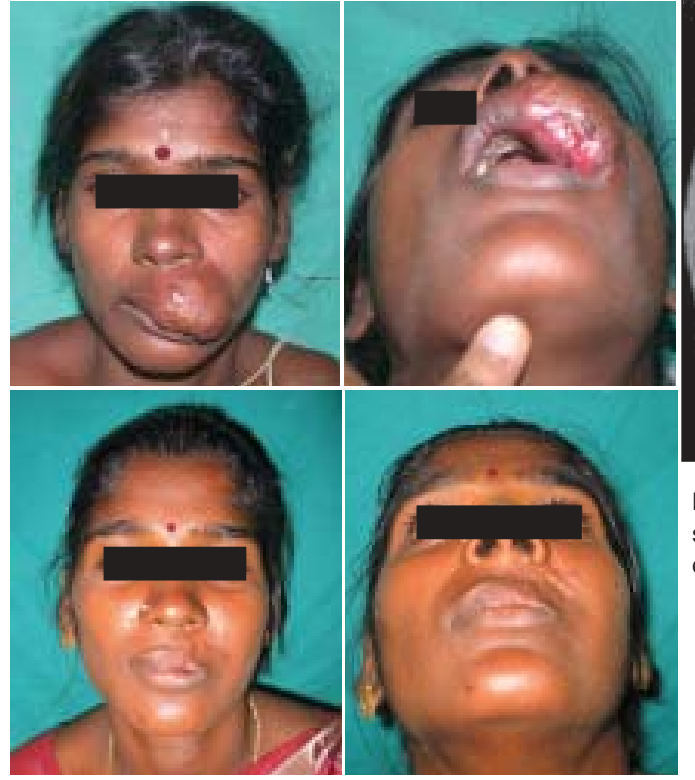

the epicenter of the AVM and is composed of arterial feeders, micro- and macro-ateriovenous fistulas and enlarged veins.

Arteriography must precede interventional therapy or surgical excision. Surgery is performed 24-72 hours after embolisation for temporary occlusion of the feeding arteries. The surgical goal is complete resection, which is sometimes very mutilating. Intraoperative frozen section of resection margin be helpful. Superselective arterial or retrograde venous embolisaiton can be palliative for control of pain, bleeding or congestive heart failure. This is indicated when surgical resection is not possible. Sclerotherapy is another radiologic approach that may be used if the feeding arteries are ligated. This needs direct puncture of nidus, in conjuction with local arterial and venous occlusion.

After combined embolisaiton/resection, the patient must be followed up for years by clinical examination, ultrasonography and/or MRI. The goal is to control the recurrence of the AVM, as cure is usually not possible.

\section{COMBINED VASCULAR MALFOMATION}

Combined malformations can be slow-flow or fast-flow; many are remembered by eponyms and exhibit overgrowth.

\section{Slow flow combined vascular malformation}

Klippel-Trenaunay syndrome is a well-known combined capillary-lymphaticovenous malformation (CLVM) associated with soft tissue/skeletal hypertrophy. CMs are multiple and present in geographic (irregular shapes) patterns over the lateral side of the extremity, buttocks and/or thorax, usually studded with hemolymphatic vesicles (CLM). The anomalous veins are prominent laterally because of insufficiency due to absent valves and deep venous anomalies. Lymphatic hypoplasia or localized lymphatic anomalies are primary defects. Limb hypertrophy can be grotesque.

Proteus syndrome is a sporadic disorder characterized by connective tissue nevi, lipomas, several unusal tumors, and disproportionate skeletal growth, in addition to ocular, pulmonary and renal abnormalities. Vascular anomalies like CM, VM, LM or combined malformations can occur in this syndrome.

In Maffuci Syndrome exophytic venous anomalies coexist with bony exostoses and enchondromatoses. These features usually do not manifest until early to midchildhood.

\section{Fast-Flow Combined Malformation}

Parkes Weber syndrome (CAVM, CLAVM) is present at birth. There is warmth, bruit and thrill over the limb and proximal trunk. The lower limb is more affected than the upper limb. MRI in young children often reveals only diffuse hypervascularity of enlarged muscles and bones. Ateriography demonstrates microscopic AV fistulae throughout the affected limb, particularly near joints.

Bannayan-Riley-Ruvalcaba syndrome is characterized by delayed motor and speech development, proximal myopathy, macrocephaly, pigmented penile macules, ileal and colonic hamartomatous polyps, subcutaneous lipomas and Hashimoto thyroiditis. They can present as 


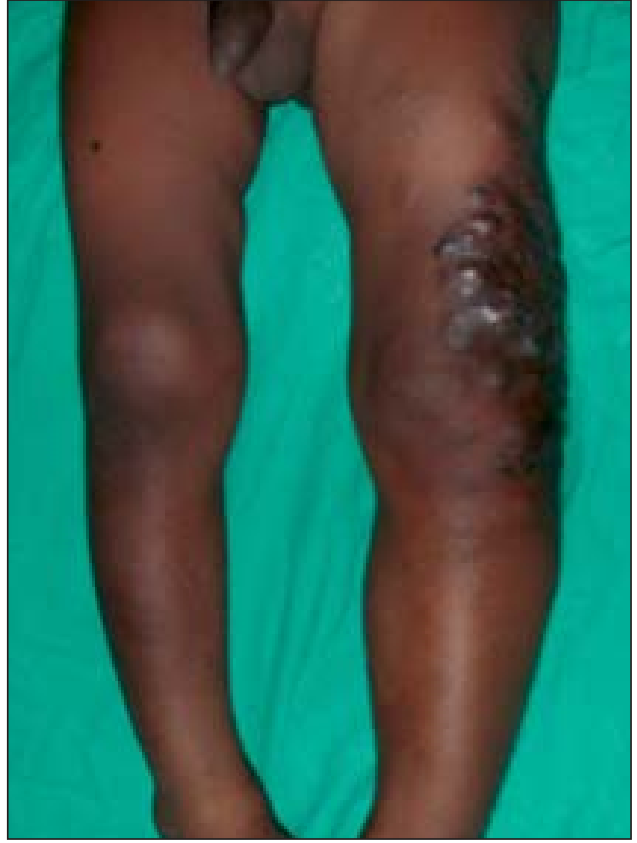

Figure 6: Combined LM, CM, and VM with hypertrophy of limb: Klipple Trenaunay Syndrome

small lesions to extensive AVMs. This is autosomal dominant disorder with the risk of developing benign or malignant neoplasia.

To conclude, it cannot be emphasized more to have a correct diagnosis for the vascular anomalies before the treatment. Proper understanding of the natural course and multidisciplinary approach is essential to tackle these complex anomalies. Management of combined malformation needs very carefully planned interdisciplinary treatment. It is difficult to cure these lesions, and the aim is to control them and treat the associated complications.

\section{ACKNOWLEDGMENT}

I am grateful to John Butler Mulliken Foundation for sponsoring my training in "Multidisciplinary Management of Vascular Malformation" at Children's Hospital, Harvard University, Boston, USA.

\section{REFERENCES}

1. Mulliken JB. Young AE. Vascular birthmarks: Hemangiomas and Malformations, Philadelphia: Saunders; 1988.

2. Mulliken JB, Fishman SJ, Burrow PE. Vascular Anomalies. Curr Prob Surg 2000;37:517.

3. Marler JJ, Mulliken JB. Vascular Anomalies, Classification, diagnosis and natural history: Facial Plast Surg Clin North Am 2001;9:495-503.

4. Boon LM, MacDonald DM, Mulliken JB. Complication of systemic corticosteroid therapy for problematic hemangiomas. Plast Reconstr Surg 2002;109:1544.

5. Katherine DH, Mulliken JB, et al. Venous malformation of skeletal muscle. Plast Reconstr Surg 110:625-1635.

6. Berenguer B, Burrows PE, Aurakowski D, Mulliken JB. Sclerotherapy of Craniofacial venous malformation: Complications and results. Plast Reconstr Surg 1999;104:1-15.

7. Berenguer B, Burrows PE, Zurakowski D, Mulliken JB. Sclerotherapy of craniofacial venous malformation: Complication and outcome: Plast Reconstr Surg 1999;104:1.

8. Kohout MP, Hansen M, Pribaz JJ, Mulliken JB. Ateriovenous malformation of head and neck: Natural history and management. Plast Reconstr Surg 1998;102:643.

\section{Annoucement}

A 'Paediatric Plastic Surgery Course' is being held in Melbourne, Australia, from 10-13 November 2005.

Presented by the Royal Children's Hospital Department of Plastic and Maxillofacial Surgery in association with the Royal Australasian College of Surgeons it will have Mr. John Reinisch, MD, FACS and Mr. Ronald Zuker, MD, FRCS(C),FACS, FAAP as Keynote Speakers. Melbourne is marvelous at any time of the year but November is summer at its very mild best. For those of you who would care to take the trip Down Under please contact Ms.

Sarah McCormick, Conference Co-ordinator at:

Course Secretariat

c/- ICMS Pty Ltd

84 Queensbridge Street

Southgate, VIC

Australia, 3006

Phone: +61 39682 0244, Fax: +61 396820288

Email: marketing@icms.com.au

For more information on the course please see: <http://www.pps2005.com> www.pps2005.com 